\title{
National Survey of Hospitalists' Experiences with Incidental Pulmonary Nodules
}

\author{
Craig A Umscheid, MD, MS',2,3,4*; Jonathan Wilen, MD5; Matthew Garin, MD'1,6; Jenna D Goldstein, MA7; \\ Tessa S Cook, MD, PhD'; Yulun Liu, PhD; ; Yong Chen, PhD ${ }^{1,4}$; Jennifer S Myers, MD ${ }^{1,3}$
}

\begin{abstract}
${ }^{1}$ University of Pennsylvania Perelman School of Medicine, Philadelphia, Pennsylvania; ${ }^{2}$ Center for Evidence-based Practice, University of Pennsylvania Health System, Philadelphia, Pennsylvania; ${ }^{3}$ Center for Healthcare Improvement and Patient Safety, University of Pennsylvania Perelman School of Medicine, Philadelphia, Pennsylvania; ${ }^{4}$ Center for Clinical Epidemiology and Biostatistics, University of Pennsylvania Perelman School of Medicine, Philadelphia, Pennsylvania; ${ }^{5}$ New York Presbyterian - Columbia University Medical Center, New York, New York; ${ }^{6}$ Corporal Michael J. Crescenz Veterans Affairs Medical Center, Philadelphia, Pennsylvania; ${ }^{7}$ Center for Quality Improvement, Society of Hospital Medicine, Philadelphia, Pennsylvania; ${ }^{8}$ Department of Clinical Sciences, University of Texas Southwestern Medical Center, Dallas, Texas.
\end{abstract}

Incidental pulmonary nodules (IPNs) are common and often require follow-up. The Fleischner Society guidelines were created to support IPN management. We developed a 14-item survey to examine hospitalists' exposure to and management of IPNs. The survey targeted attendees of the 2016 Society of Hospital Medicine (SHM) annual conference. We recruited 174 attendees. In total, 82\% were identified as hospitalist physicians and $7 \%$ as advanced practice providers; $63 \%$ practiced for $>5$ years and $62 \%$ supervised trainees. All reported seeing $\geq 1$ IPN case in the past six months, with $39 \%$ seeing three to five cases and $39 \%$ seeing six or more cases.
Notwithstanding, 42\% were unfamiliar with the Fleischner Society guidelines. When determining the IPN follow-up, $83 \%$ used radiology report recommendations, $64 \%$ consulted national or international guidelines, and $34 \%$ contacted radiologists; $34 \%$ agreed that determining the follow-up was challenging; only $15 \%$ reported availability of automated tracking systems. In conclusion, despite frequent IPN exposure, hospitalists are frequently unaware of the Fleischner Society guidelines and rely on radiologists' recommendations. Journal of Hospital Medicine 2019;14:353-356. Published online first February 20, 2019. (C) 2019 Society of Hospital Medicine

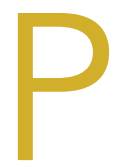

ulmonary nodules are common, and their identification is increasing as a result of the use of more sensitive chest imaging modalities. ${ }^{1}$ Pulmonary nodules are defined on imaging as small $(\leq 30 \mathrm{~mm})$, well-defined lesions, completely surrounded by pulmonary parenchyma. ${ }^{2}$ Most of the pulmonary nodules detected incidentally (ie, in asymptomatic patients outside the context of chest CT screening for lung cancer) are benign. ${ }^{1}$ Lesions $>30 \mathrm{~mm}$ are defined as masses and have higher risks of malignancy. ${ }^{2}$

Because the majority of patients will not benefit from the identification of incidental pulmonary nodules (IPNs), improving the benefits and minimizing the harms of IPN follow-up are critical. The Fleischner Society ${ }^{3}$ published their first guideline on the management of solid IPNs in 2005, ${ }^{4}$ which was supplemented in 2013 with specific guidance for the management of subsolid IPNs. ${ }^{5}$ In 2017, both guidelines were combined in a single update. ${ }^{6}$ The Fleischner Society recommendations for imaging surveillance and tissue sampling are based on nodule type (solid vs subsolid), number (single vs multiple), size, appearance, and patient risk for malignancy.

For IPNs identified in the hospital, management may be particularly challenging. For one, the provider initially ordering the

*Corresponding Author: Craig A. Umscheid, MD, MSCE; E-mail: craig.umscheid@gmail.com; Telephone: 215-349-8098.

Additional Supporting Information may be found in the online version of this article.

Received: June 15, 2018; Revised: October 2, 2018;

Accepted: October 18, 2018

() 2019 Society of Hospital Medicine DOI 10.12788/jhm.3115 chest imaging may not be the provider coordinating the patient's discharge, leading to a lack of knowledge that the IPN even exists. The hospitalist to primary care provider (PCP) handoff may also have vulnerabilities, including the lack of inclusion of the IPN follow-up in the discharge summary and the nonreceipt of the discharge summary by the PCP. Moreover, because a patient's acute medical problems often take precedence during a hospitalization, inpatients may not even be made aware of identified IPNs and the need for follow-up. Thus, the absence of standardized approaches to managing IPNs is a threat to patient safety, as well as a legal liability for providers and their institutions.

To better understand the current state of IPN management in our own institution, we examined the management of IPNs identified by chest computed tomographies (CTs) performed for inpatients on our general medicine services over a two-year period. ${ }^{7}$ Among the 50 inpatients identified with IPNs requiring follow-up, 78\% had no follow-up imaging documented. Moreover, $40 \%$ had no mention of the IPN in their hospital summary or discharge instructions.

To inform our approach to addressing this challenge, we sought to examine the practices of hospitalist physicians nationally regarding the management of IPNs, including hospitalists' familiarity with the Fleischner Society guidelines.

\section{METHODS}

We developed a 14-item survey to assess hospitalists' exposure to and management of IPNs. The survey targeted attendees of the 2016 Society of Hospital Medicine (SHM) annual conference and was available for completion on a tablet at the conference 
TABLE 1. Demographics of Respondents of a National Survey of Hospitalists' Experience with Incidental Pulmonary Nodules

\begin{tabular}{|c|c|c|}
\hline Survey Item & Response Choices & n (\%) \\
\hline \multirow[t]{4}{*}{ What is your role in patient care? ${ }^{\text {a }}$} & Hospitalist Physician & $138(82 \%)$ \\
\hline & Advanced Practitioner (NP, PA) & $12(7 \%)$ \\
\hline & Non-Hospitalist General Internist (I care for inpatients and outpatients) & $5(3 \%)$ \\
\hline & General Internal Medicine Fellow & $6(3 \%)$ \\
\hline \multirow[t]{5}{*}{ How many years have you been in practice? } & $<5$ years & $63(37 \%)$ \\
\hline & $5-9$ years & $36(21 \%)$ \\
\hline & $10-15$ years & $38(22 \%)$ \\
\hline & $16-20$ years & $11(7 \%)$ \\
\hline & $>20$ years & $22(13 \%)$ \\
\hline \multirow[t]{4}{*}{ What is your current practice setting? (Please specify all that apply)* } & University/Teaching hospital & $86(49 \%)$ \\
\hline & Private hospital & $21(12 \%)$ \\
\hline & Veterans Affairs hospital & $9(5 \%)$ \\
\hline & Community hospital & $65(37 \%)$ \\
\hline \multirow[t]{4}{*}{ What is your specialty? } & Internal Medicine & $147(87 \%)$ \\
\hline & Family Medicine & $19(11 \%)$ \\
\hline & Pediatrics & $0(0 \%)$ \\
\hline & Obstetrics - Gynecology & $0(0 \%)$ \\
\hline \multirow[t]{2}{*}{ Do you supervise medical students, residents, or fellows in clinical service? } & Yes & $105(62 \%)$ \\
\hline & No & $65(38 \%)$ \\
\hline \multirow[t]{6}{*}{ Which one option best describes your current practice location? } & Northeast & $52(31 \%)$ \\
\hline & Midwest & $34(20 \%)$ \\
\hline & Southeast & $35(21 \%)$ \\
\hline & Southwest & $14(8 \%)$ \\
\hline & West & $19(11 \%)$ \\
\hline & Pacific Northwest & $13(8 \%)$ \\
\hline
\end{tabular}

"This survey item also included "Other, please specify" as a response choice.

Abbreviations; NP, nurse practitioner; PA, physician assistant.

registration desk, the SHM kiosk in the exhibit hall, and at the entrance and exit of the morning plenary sessions. Following the annual conference, the survey was e-mailed to conference attendees, with one follow-up e-mailed to nonresponders.

Analyses were descriptive and included proportions for categorical variables and median and mean values and standard deviations for continuous variables. In addition, we examined the association between survey items and a response of "yes" to the question "Are you familiar with the Fleischner Society guidelines for the management of incidental pulmonary nodules?"

Associations between familiarity with the Fleischner Society guidelines and survey items were examined using Pearson's chi-square test for categorical variables, Fisher's exact test for categorical variables with small sample sizes, the Cochran-Armitage test for trend for ordinal variables, and the t-test for continuous variables. The associations between categorical items were measured by odds ratios with $95 \%$ confidence intervals. Statistical tests were two-sided using a $P=.05$ level for statistical significance. All analyses were performed using $R$ version 3.4.4 (R Foundation for Statistical Computing, Vienna, Austria), with the R packages MASS, stats, and Publish. Institutional review board exemption was granted.

\section{RESULTS}

We received 174 responses from a total of 3,954 conference attendees. The majority were identified as hospitalist physicians, and most of them were internists (Table 1). About half practiced at a university or a teaching hospital, and more than half supervised trainees and practiced for more than five years. Respondents were involved in direct patient care (whether a teaching or a nonteaching service) for a median of 28 weeks annually (mean 31.2 weeks, standard deviation 13.5), and practice regions were geographically diverse. All respondents reported seeing at least one IPN case in the past six months, with most seeing three or more cases (Table 2). Despite this exposure, $42 \%$ were unfamiliar with the Fleischner Society guidelines. When determining the need for IPN follow-up, most of them utilized radiology report recommendations or consulted national or international guidelines, and a third spoke with radiologists directly. About a third agreed that determining the need for follow-up was challenging, with 39\% citing patient factors (eg, lack of insurance, poor access to healthcare), and $30 \%$ citing scheduling of follow-up imaging. Few reported the availability of an automated tracking system at their institution, although most of them desired automatic notifications of results requiring follow-up. 
TABLE 2. Results of a National Survey of Hospitalists' Experience with Incidental Pulmonary Nodules

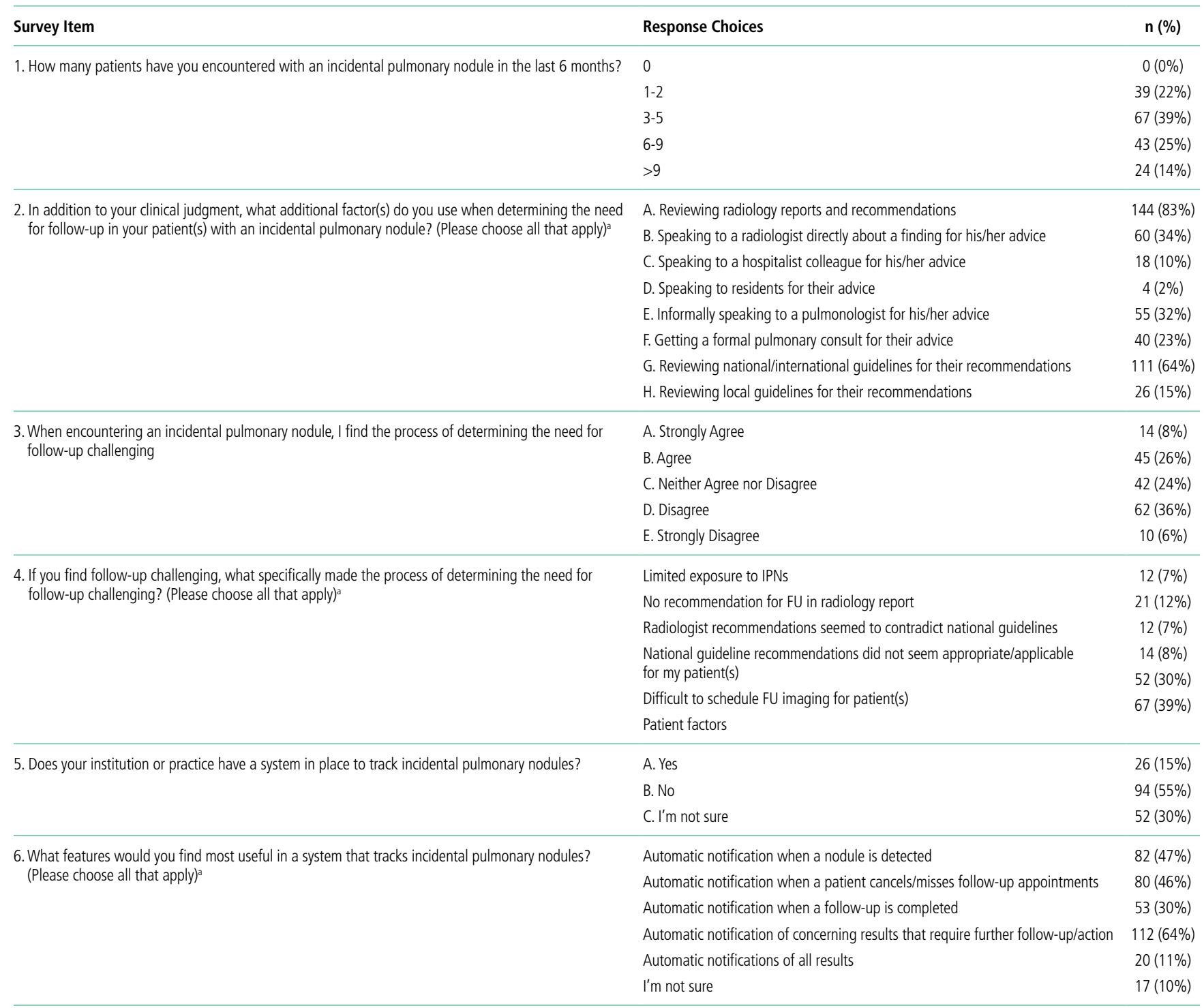

"This survey item also included "Other, please specify" as a response choice.

Abbreviations: FU, follow-up; IPN, incidental pulmonary nodule.

Unadjusted analyses revealed that supervision of trainees and seeing more IPN cases significantly increased the odds of a survey respondent being familiar with the Fleischner Society guidelines (OR 1.96, 95\% Cl 1.04-3.68, $P=.05$, and OR 1.55, 95\% Cl 1.12-2.18, $P=.008$, respectively; Supplementary Table 1).

\section{DISCUSSION}

To our knowledge, the survey reported here is the first to examine hospitalists' knowledge of the Fleischner Society guidelines and their approach to management of IPNs. Although our data suggest that hospitalists are less familiar with the Fleischner Society recommendations than pulmonologists ${ }^{8}$ and radiologists, ${ }^{8-10}$ the majority of hospitalists in our study rely on radiology report recommendations to inform follow-up. This suggests that embedding the Fleischner Society recommendations into ra- diology reports is an effective method to promote adherence to these recommendations, which has been demonstrated in previous research..$^{11-13}$ Our study also suggests that hospitalists with more IPN exposure and those who supervise trainees are more likely to be aware of the Fleischner Society recommendations, which is similar to findings from studies examining radiologists and pulmonologists. ${ }^{8-9}$

Our findings highlight other opportunities for quality improvement in IPN management. Almost a quarter of hospitalists reported formally consulting pulmonologists for IPN management. Hospitalist groups wishing to improve value could partner with their radiology departments and embed the Fleischner Society recommendations into their imaging reports to potentially reduce unnecessary pulmonary consultations. Among the 59 hospitalists who agreed that IPN management was challenging, a majority 
cited the scheduling process (30\%) as a barrier. Redesigning the scheduling process for follow-up imaging could be a focus in local efforts to improve IPN management. Strengthening communication between hospitalists and PCPs may provide additional opportunities for improved IPN follow-up, given the centrality of PCPs to ensuring such follow-up. This might include enhancing direct communication between hospitalists and PCPs for high-risk patients, or creating systems to ensure robust indirect communication, such as the implementation of standardized discharge summaries that uniformly include essential follow-up information.

At our institution, given the large volume of high-risk patients and imaging performed, and the available resources, we have established an IPN consult team to improve follow-up for inpatients with IPNs identified by chest CTs on Medicine services. The team includes a nurse practitioner (NP) and a pulmonologist who consult by default, to notify patients of their findings and recommended follow-up, and communicate results to their PCPs. The IPN consult team also sees patients for follow-up in the ambulatory IPN clinic. This initiative has addressed the most frequently cited challenges identified in our nationwide hospitalist survey by taking the communication and follow-up out of the hospitalists' hands. To ensure identification of all IPNs by the NP, our radiology department has created a structured template for radiology attendings to document follow-up for all chest CTs reviewed based on the Fleischner Society guidelines. Compliance with use of the template by radiologists is followed monthly. After a run-in period, almost $100 \%$ of chest CT reports use the structured template, consistent with published findings from similar initiatives, ${ }^{14}$ and $100 \%$ of patients with new IPNs identified on the inpatient Medicine services have had an IPN consult.

The major limitation of our survey study is the response rate. It is difficult to determine in what direction this could bias our results, as those with and without experience in managing IPNs may have been equally likely to complete the survey. Despite the low response rate, our sample targeted the general cohort of conference attendees (rather than specific forums such as audiences interested in quality or imaging), and the descriptive characteristics of our convenience sample align well with the overall conference attendee demographics (eg, conference attendees were $77 \%$ hospitalist attendings and $9 \%$ advanced practice providers, as compared with $82 \%$ and $7 \%$ of survey respondents, respectively), suggesting that our respondents were representative of conference attendees as a whole.

Next steps for this work at our institution include developing systems to ensure appropriate follow-up for those with IPNs identified on chest CTs performed for Medicine outpatients. In addition, our institution is collaborating on a national study to compare outcomes resulting from following the traditional Fleischner Society recommendations compared to the new 2017 recommendations, which recommend more lenient follow-up. ${ }^{15}$

\section{Acknowledgments}

The authors acknowledge Vivek Ahya, Eduardo Barbosa Jr., Tammy Tursi, and Anil Vachani for their leadership of the local quality improvement initiatives described in our Discussion, namely, the development and implementation of the structured templates for radiology reports and the incidental pulmonary nodule consult team.

Disclosures: Dr. Cook reports relevant financial activity outside the submitted work, including royalties from Osler Institute and grants from ACRIN and Beryl Institute. All other authors report no potential conflicts of interest relevant to this study. There was no financial support for this study.

Previous Presentations: Presented as a poster at the 2017 Society of Hospital Medicine Annual Conference, Las Vegas, NV: Wilen J, Garin M, Umscheid CA, Cook TS, Myers JS. Follow-up of incidental pulmonary nodules: a survey of hospitalists nationwide [abstract]. Journal of Hospital Medicine. 2017; 12 (Suppl 2). Available at: https://www.shmabstracts.com/abstract/follow-up-of-incidental-pulmonary-nodules-a-survey-of-hospitalists-nationwide/. Accessed March 18, 2018.

\section{References}

1. Ost D, Fein AM, Feinsilver SH. Clinical Practice: the solitary pulmonary nodule. N Engl J Med. 2003;348(25):2535-2542. doi: 10.1056/NEJMcp012290

2. Tuddenham WJ. Glossary of terms for thoracic radiology: recommendations of the Nomenclature Committee of the Fleischner Society. AJR Am J Roentgenol. 1984;143(3):509-517.

3. Janower ML. A brief history of the Fleischner Society. J Thorac Imaging. 2010;25(1):27-28. doi: 10.1097/RTI.0b013e3181cc4cee.

4. Macmahon H, Austin JH, Gamsu G, et al. Guidelines for management of small pulmonary nodules detected on CT scans: a statement from the Fleischner Society. Radiology. 2005;237(2):395-400. doi: 10.1148/radiol.2372041887.

5. Naidich DP, Bankier AA, MacMahon $H$, et al. Recommendations for the management of subsolid pulmonary nodules detected at CT: a statement from the Fleischner Society. Radiology. 2013;266(1):304-317. doi: 10.1148/ radiol. 12120628

6. MacMahon H, Naidich DP, Goo JM, et al. Guidelines for management of incidental pulmonary nodules detected on CT images: from the Fleischner Society 2017. Radiology.2017;284(1):228-243. doi: 10.1148/radiol.2017161659.

7. Garin M, Soran C, Cook T, Ferguson M, Day S, Myers JS. Communication and follow-up of incidental lung nodules found on chest CT in a hospitalized and ambulatory patient population. J Hosp Med. 2014:9(2). Available at: https://www.shmabstracts.com/abstract/communication-and-followup-of-incidental-lung-nodules-found-on-chest-ct-in-a-hospitalized-and-ambulatory-patient-population/ Accessed June 14, 2018.

8. Mets OM, de Jong PA, Chung K, Lammers JWJ, van Ginneken B, Schaefer-Prokop CM. Fleischner recommendations for the management of subsolid pulmonary nodules: high awareness but limited conformance - a survey study. Eur Radiol. 2016;26:3840-3849. doi: 10.1007/s00330-016-4249-y.

9. Eisenberg RL, Bankier AA, Boiselle PM. Compliance with Fleischner Society Guidelines for management of small lung nodules: a survey of 834 radiologists. Radiology. 2010;255(1):218-224. doi: 10.1148/radiol.09091556.

10. Eisenberg RL. Ways to improve radiologists' adherence to Fleischner society guidelines for management of pulmonary nodules. J Am Coll Radiol. 2013;10(6):439-441. doi: 10.1016/j.jacr.2012.10.001.

11. Blagev DP, Lloyd JF, Conner K, et al. Follow-up of incidental pulmonary nodules and the radiology report. J Am Coll Radiol. 2014;11(4):378-383. doi: 10.1016/j.jacr.2013.08.003.

12. Woloshin S, Schwartz LM, Dann E, Black WC. Using radiology reports to encourage evidence-based practice in the evaluation of small, incidentally detected pulmonary nodules: a preliminary study. Ann Am Thorac Soc. 2014;11(2):211-214. doi: 10.1513/AnnalsATS.201307-242BC.

13. McDonald JS, Koo CW, White D, Hartman TE, Bender CE, Sykes AMG. Addition of the Fleischner Society Guidelines to chest CT examination interpretive reports improves adherence to recommended follow-up care for incidental pulmonary nodules. Acad Radiol. 2017;24(3):337-344. doi: 10.1016/j. acra.2016.08.026.

14. Zygmont ME, Shekhani H, Kerchberger JM, Johnson JO, Hanna TN. Pointof-Care reference materials increase practice compliance with societal guidelines for incidental findings in emergency imaging. J Am Coll Radiol. 2016;13(12):1494-1500. doi: 10.1016/j.jacr.2016.07.032.

15. Patient-Centered Outcomes Research Institute Portfolio of Funded Projects. Available at: https://www.pcori.org/research-results/2015/pragmatic-trial-more-versus-less-intensive-strategies-active-surveillance\#/. Accessed May 22, 2018. 